\title{
From local to global importance measures of uncertainty propagation in composite structures
}

\author{
Carlos Conceição António, Luísa N. Hoffbauer
}

\begin{abstract}
The influence of uncertainties of input parameters on output response of composite structures is investigated in this paper. In particular, the effects of deviations in mechanical properties, ply angles, ply thickness and on applied loads are studied. The uncertainty propagation and the importance measure of input parameters are analysed using three different approaches: a first-order local method, a Global Sensitivity Analysis (GSA) supported by a variance-based method and an extension of local variance to estimate the global variance over the domain of inputs.

Sample results are shown for a shell composite laminated structure built with different composite systems including multi-materials. The importance measures of input parameters on structural response based on numerical results are established and discussed as a function of the anisotropy of composite materials. Needs for global variance methods are discussed by comparing the results obtained from different proposed methodologies. The objective of this paper is to contribute for the use of GSA techniques together with low expensive local importance measures.
\end{abstract}

Keywords: Uncertainty; Composite structures; Local importance measure; Global variance-based; Global approach

\section{Introduction}

Structural applications of laminate composite materials have increased due to their excellent specific stiffness and low weight together with reduced energy consumption. Variations of manufacturing process parameters and environment aspects may affect quality and performance of the product with consequences to its structural behaviour. Robust design optimisation (RDO) searches for safe structural systems with minimal variability in the response when subjected to uncertainties at the input parameters. The robust design procedure pursues two main objectives: keeping at an acceptable level the variations of the structural performance and controlling the magnitude of uncertainty at the input parameters.
Not many researchers have developed models for robust design of composite structures considering uncertainty at input parameters. Oh and Librescu [1] address the problem of alleviating the effects of unavoidable parameter uncertainties for free vibration of composite cantilevers under uncertainties at layer thickness, elastic constants and ply angle. The structural tailoring technique was applied to design laminated composite structures identifying the less sensitive performance properties to uncertainties of the input parameters. Noor et al. [2] studied the variability of non-linear response of stiffened composite panels due to variations in geometric and material parameters. Singh et al. [3] investigated the influence of variations of material properties on the elastic stability of laminated composite panels. Adali et al. [4] developed a model aimed at the optimal design of composite laminates under buckling load uncertainty. In this model loads belong to a given uncertainty domain. Walker and Hamilton [5] described a procedure to design symmetric laminates for maximum buckling 
load under manufacturing uncertainty in ply-angle design variable. Gumbert and Newman [6] analyse the effect of geometric uncertainty in shape parameters in a 3-D flexible wing. Yang et al. [7] investigated the stochastic bending response of compositionally graded plates with uncertainties of low variability in thermal and mechanical properties, volume fraction and load intensity. Using a Monte Carlo analysis Ramroth et al. [8] estimated the uncertainty in thermal behaviour due to changes in thermo-mechanical input parameters.

An important task of robust design research is the development of uncertainty assessment methods for structural applications. Although several methods have been presented for uncertainty assessment $[9,10]$ it was not proven their efficiency in particular when applied to composite structures. Most of the researchers referred in applications with composite structures [1-8] used local importance measures centred on mean values of input parameters and needs for Global Sensitivity Analysis (GSA) on the uncertainty response of composite structures were unexplored staying an open issue. The objective of this paper is to contribute for the use of GSA techniques together with low expensive local importance measures.

The paper is organised as follows: first, the modelling of composite shell structures is summarised and output response functions are defined. Second, a local method for uncertainty propagation on output responses is established. This method is based on first-order Taylor expansion and uses an adjoint procedure for sensitivity analysis. The coefficient of variation and local variance decomposition technique are used. Third, a Global Sensitivity Analysis (GSA) is presented namely a variance-based method is developed. The first-order sensitivity index proposed by Sobol [11] is adopted together with a methodology based on Monte Carlo simulation for GSA evaluation. Fourth, an approach based on local uncertainty propagation evaluation is extended over the whole domain of input parameters. The objective is to estimate the global variance and this way overcoming the expensive costs associated with variance-based methods. Finally, results obtained from the uncertainty analysis are presented and discussed. Comparison of approaches is established particularly for the case of uncertainties in ply mechanical properties of composite laminates.

\section{Response of composite structures}

\subsection{FEM formulation}

The structural analysis of laminated composite structures is based on the shell finite element model developed by Ahmad [12] and further improvements [13]. This shell element is obtained from a 3-D finite element using a degenerative procedure. It is an isoparametric element with eight nodes and five freedom degrees per node based on the Mindlin shell theory. The shell consists of a number of perfectly bonded plies. Each individual ply are assumed homogeneous and anisotropic.

The displacement vector at each $k$ th node is

$\delta_{k}=\left(u_{k}, v_{k}, w_{k}, \beta_{1 k}, \beta_{2 k}\right)$

with three independent translations $u_{k}, v_{k}, w_{k}$ and two independent rotations $\beta_{1 k}, \beta_{2 k}$. The displacement vector of the element is

$\boldsymbol{\delta}^{\mathrm{e}}=\left(\delta_{1}, \ldots, \delta_{k}, \ldots, \delta_{n}\right)$

being $n$ the number of degrees of freedom of the element. The displacement field in the $i$ th element can be expressed as

$\mathbf{u}_{i}=\sum_{k=1}^{n}\left(N_{k}(\xi, \eta) \mathbf{u}_{i k}^{\mathrm{mid}}+N_{k}(\xi, \eta) \frac{1}{2} \zeta h_{k}\left[\overline{\mathbf{v}}_{1 k} \overline{\mathbf{v}}_{2 k}\right]\left(\beta_{1 k}, \beta_{2 k}\right)^{\mathrm{T}}\right)$

where $N_{k}(\xi, \eta)$ are the shape functions, $(\xi, \eta, \zeta)$ is the local curvilinear coordinate system being $\xi$ and $\eta$ defined in the middle plan of the shell element and $\zeta$ is the coordinate associated to the thickness direction. In Eq. (3) $\mathbf{u}_{i k}^{\text {mid }}$ is the displacement vector of the $k$ th node referred to the middle surface of the shell, $h_{k}$ is the thickness defined by upper and lower surfaces of the shell at the $k$ th node and $\overline{\mathbf{v}}_{1 k}$ and $\overline{\mathbf{v}}_{2 k}$ are the cosines of the nodal coordinate system associated to the middle surface.

The stress and strain are referred to local coordinate system $\left(X^{\prime}, Y^{\prime}, Z^{\prime}\right)$ related with the surface $\zeta$ taken as constant. The strain vector is

$$
\boldsymbol{\varepsilon}^{\prime}=\left\{\begin{array}{c}
\varepsilon_{x}^{\prime} \\
\varepsilon_{y}^{\prime} \\
\gamma_{x y}^{\prime} \\
\gamma_{x z}^{\prime} \\
\gamma_{y z}^{\prime}
\end{array}\right\}=\left\{\begin{array}{c}
\frac{\partial u}{\partial x^{\prime}} \\
\frac{\partial v}{\partial x^{\prime}} \\
\frac{\partial u}{\partial y^{\prime}}+\frac{\partial v}{\partial x^{\prime}} \\
\frac{\partial u}{\partial z^{\prime}}+\frac{\partial w}{\partial x^{\prime}} \\
\frac{\partial w}{\partial y^{\prime}}+\frac{\partial v}{\partial z^{\prime}}
\end{array}\right\}
$$

In general it can be written the following strain-displacement relationship:

$\boldsymbol{\varepsilon}^{\prime}=\mathbf{B u}$

where $\mathbf{B}$ is the strain matrix and $\mathbf{u}$ is the displacement vector.

The stress-strain constitutive relations can be written as

$\boldsymbol{\sigma}^{\prime}=\mathbf{D}^{\prime} \boldsymbol{\varepsilon}^{\prime}$

being the stress and strain vectors defined as

$\boldsymbol{\sigma}^{\prime}=\left(s_{x^{\prime}}, s_{y^{\prime}}, s_{x^{\prime} y^{\prime}}, s_{x^{\prime} z^{\prime}}, s_{y^{\prime} z^{\prime}}\right)$

$\boldsymbol{\varepsilon}^{\prime}=\left(\varepsilon_{x^{\prime}}, \varepsilon_{y^{\prime}}, \gamma_{x^{\prime} y^{\prime}}, \gamma_{x^{\prime} z^{\prime}}, \gamma_{y^{\prime} z^{\prime}}\right)$

and the matrix $\mathbf{D}^{\prime}$ with the elastic constants is for each ply

$\mathbf{D}^{\prime}=\mathbf{T}^{\mathrm{T}} \mathbf{D} \mathbf{T}$

$\mathbf{D}=\left[\begin{array}{cc}\mathbf{D}_{1} & \mathbf{0} \\ \mathbf{0} & \mathbf{D}_{2}\end{array}\right]$

with 
$\mathbf{D}_{1}=\left[\begin{array}{ccc}\frac{E_{1}}{1-v_{12} v_{21}} & \frac{v_{21} E_{2}}{1-v_{12} v_{21}} & 0 \\ \frac{v_{12} E_{2}}{1-v_{12} v_{21}} & \frac{E_{2}}{1-v_{12} v_{21}} & 0 \\ 0 & 0 & G_{12}\end{array}\right]$
$\mathbf{D}_{2}=\left[\begin{array}{cc}k_{1} G_{13} & 0 \\ 0 & k_{2} G_{23}\end{array}\right]$

where the elastic constants of orthotropic ply are the longitudinal elastic modulus $E_{1}$, the transversal elastic modulus $E_{2}$, the in-plane shear modulus $G_{12}$, the out-of-plane shear modulus $G_{13}$ and $G_{23}$ and the in-plane Poisson's ratio $v_{12}$. The constants $k_{1}, k_{2}$ are shear correction factors [13].

In this work it is considered the linear elastic behaviour of composite structures with the equilibrium equation set established as

$\mathbf{K u}=\mathbf{F}$

where $\mathbf{K}$ is the stiffness matrix with each element calculated as

$\mathbf{K}^{\mathrm{e}}=\int_{V^{\mathrm{e}}} \mathbf{B}^{\mathrm{T}} \mathbf{D}^{\prime} \mathbf{B} \mathrm{d} V^{\mathrm{e}}$

being $\mathbf{u}$ the displacement vector and $\mathbf{F}$ the applied external loads vector. Other details of the finite element formulation are reported in references $[12,13]$.

\subsection{Response functions}

Consider the system response of the composite structure under static loading represented by the function $\Psi(X)$ with $p$ input parameters denoted as $\mathbf{X}=\left(X_{1}, \ldots, X_{p}\right)$. In the present work the system response is obtained as a function of the displacement and stress obtained after the structural analysis and it can be written as

$\boldsymbol{\Psi}(\mathbf{X})=[\bar{u}(\mathbf{X}) \bar{R}(\mathbf{X})]^{\mathrm{T}}$

where $\bar{u}$ is the absolute value of maximum displacement and $\bar{R}$ the critical Tsai number. The maximum displacement on the structure is defined as,

$\bar{u}=\operatorname{Max}\left(\left|u_{1}\right|, \ldots,\left|u_{N_{\mathrm{d}}}\right|\right)$

being $N_{\mathrm{d}}$ the total number of displacements. Defining the Tsai number $R_{k}$ as a strength/stress ratio [14] it can be introduced in the interactive quadratic failure criterion of Tsai-Wu and calculated at the $k$ th point of the structure where the stress vector is evaluated, by solving the equation

$1-\left(F_{i j} s_{i} s_{j}\right) R_{k}^{2}+\left(F_{i} s_{i}\right) R_{k}=0$

being $s_{i}$ components of the stress vector, $F_{i j}$ and $F_{i}$ the strength parameters associated with unidirectional reinforced laminate defined from the macro-mechanical point of view [14]. Since the safe region is related to $R_{k}>1$, the critical Tsai number can be established as

$\bar{R}=\operatorname{Min}\left(R_{1}, \ldots, R_{k}, \ldots, R_{N_{\mathrm{s}}}\right)$

where $N_{\mathrm{s}}$ is the total number of points where the stress vector is evaluated.

\section{Local importance measure for uncertainty analysis}

The proposed approach is based on first-order approximation in the neighbourhood of nominal values of the input parameter vector $\mathbf{X}^{0}[15]$ and is known as differential analysis [9]. The first-order Taylor expansion of the structural response function $\Psi(\mathbf{X})$ around the nominal values $\mathbf{X}^{0}$ is defined as:

$\boldsymbol{\Psi}(\mathbf{X})=\boldsymbol{\Psi}\left(\mathbf{X}^{0}\right)+\delta \boldsymbol{\Psi} \cong \boldsymbol{\Psi}\left(\mathbf{X}^{0}\right)+\mathbf{S} \delta \mathbf{X}$

where $\mathbf{S}$ is a rectangular matrix of order $2 \times n$ with components representing the sensitivity of the $j$ th response to the $i$ th system parameter. Eq. (19) can be written as

$$
\begin{aligned}
\boldsymbol{\Psi}(\mathbf{X})= & {\left[\begin{array}{l}
\bar{u}(\mathbf{X}) \\
\bar{R}(\mathbf{X})
\end{array}\right] \cong\left[\begin{array}{c}
\bar{u}\left(\mathbf{X}^{0}\right) \\
\bar{R}\left(\mathbf{X}^{0}\right)
\end{array}\right] } \\
& +\left[\begin{array}{ccc}
\frac{\partial \bar{u}}{\partial X_{1}}\left(\mathbf{X}^{0}\right) & \cdots & \frac{\partial \bar{u}}{\partial X_{n}}\left(\mathbf{X}^{0}\right) \\
\frac{\partial \bar{R}}{\partial X_{1}}\left(\mathbf{X}^{0}\right) & \cdots & \frac{\partial \bar{R}}{\partial X_{n}}\left(\mathbf{X}^{0}\right)
\end{array}\right]\left[\begin{array}{c}
\left(X_{1}-X_{1}^{0}\right) \\
\vdots \\
\left(X_{n}-X_{n}^{0}\right)
\end{array}\right]
\end{aligned}
$$

The expectation of $\boldsymbol{\Psi}$ is obtained using Eq. (20):

$$
\begin{aligned}
E\langle\boldsymbol{\Psi}(\mathbf{X})\rangle= & {\left[\begin{array}{c}
E\langle\bar{u}(\mathbf{X})\rangle \\
E\langle\bar{R}(\mathbf{X})\rangle
\end{array}\right] \cong\left[\begin{array}{c}
\bar{u}\left(\mathbf{X}^{0}\right) \\
\bar{R}\left(\mathbf{X}^{0}\right)
\end{array}\right] } \\
& +\left[\begin{array}{ccc}
\frac{\partial \bar{u}}{\partial X_{1}}\left(\mathbf{X}^{0}\right) & \cdots & \frac{\partial \bar{u}}{\partial X_{n}}\left(\mathbf{X}^{0}\right) \\
\frac{\partial \bar{R}}{\partial X_{1}}\left(\mathbf{X}^{0}\right) & \cdots & \frac{\partial \bar{R}}{\partial X_{n}}\left(\mathbf{X}^{0}\right)
\end{array}\right]\left[\begin{array}{c}
E\left\langle X_{1}-X_{1}^{0}\right\rangle \\
\vdots \\
E\left\langle X_{1}-X_{1}^{0}\right\rangle
\end{array}\right]
\end{aligned}
$$

or using (20) in close form as

$E\langle\boldsymbol{\Psi}(\mathbf{X})\rangle \cong \boldsymbol{\Psi}\left(\mathbf{X}^{0}\right)+\mathbf{S} E\left\langle\mathbf{X}-\mathbf{X}^{0}\right\rangle$

being $\mathbf{S}$ the sensitivity matrix and finally:

$E\langle\boldsymbol{\Psi}(\mathbf{X})\rangle \cong \boldsymbol{\Psi}\left(\mathbf{X}^{0}\right)$

The variance-covariance matrix $\mathbf{C}_{\boldsymbol{\Psi}}$ for $\boldsymbol{\Psi}$ is defined as

$\mathbf{C}_{\boldsymbol{\Psi}}=\left[\begin{array}{cc}\operatorname{var}(\bar{u}) & \operatorname{cov}(\bar{R}, \bar{u}) \\ \operatorname{cov}(\bar{u}, \bar{R}) & \operatorname{var}(\bar{R})\end{array}\right]$

that by definition is

$\mathbf{C}_{\boldsymbol{\Psi}} \cong E\left\langle\left(\boldsymbol{\Psi}(\mathbf{X})-\boldsymbol{\Psi}\left(\mathbf{X}^{0}\right)\right)\left(\boldsymbol{\Psi}(\mathbf{X})-\boldsymbol{\Psi}\left(\mathbf{X}^{0}\right)^{\mathrm{T}}\right\rangle\right.$

Using Eq. (20) the variance-covariance matrix is obtained [15] as follows:

$\mathbf{C}_{\boldsymbol{\Psi}} \cong \mathbf{S C}_{x} \mathbf{S}^{\mathrm{T}}$

where $\mathbf{C}_{x}$ is the covariance matrix for parameters $\left(x_{1}, \ldots, x_{n}\right)$ with components defined as

$\left(\mathbf{C}_{x}\right)_{i j}=\left\{\begin{array}{l}\operatorname{cov}\left(x_{i}, x_{j}\right)=\rho_{i j} \sigma_{i} \sigma_{j}, \quad \rho_{i j} \equiv \text { correlation coefficient } \\ i \neq j, \\ \operatorname{var}\left(x_{i}\right)=\sigma_{i}^{2}, \quad i=j\end{array}\right.$

Eq. (26) is known as the uncertainty propagation equation. Corresponding equations for the propagation of higher- 
order moments become very complex and are not considered in this work. From Eq. (26) for the propagation of uncertainties it is observed the dependence of the covariance matrix $\mathbf{C}_{\boldsymbol{\Psi}}$ relatively to the sensitivity matrix $\mathbf{S}$.

The components of the sensitivity matrix $\mathbf{S}$ are total derivatives evaluated using the adjoint sensitivity analysis procedure. Considering the implicit and the explicit relationship between the functional response and the input parameters the adjoint variable method is based on two steps. For a given functional $\Psi_{m}(\mathbf{x})=\Psi_{m}\left(X_{1}, \ldots, X_{n}\right)$ the proposed adjoint procedure follows:

1. Considering the independence of $\mathbf{F}$ to the displacements $\mathbf{u}$ in equilibrium Eq. (13), the adjoint set of equations is obtained

$\mathbf{K}(\mathbf{X}) \phi=\frac{\partial \Psi_{m}(\mathbf{u}, \mathbf{X})}{\partial \mathbf{u}}$

2. Get the sensitivities from the equation,

$\frac{\mathrm{d} \Psi_{m}}{\mathrm{~d} \mathbf{X}}=\frac{\partial \Psi_{m}(\mathbf{u}, \mathbf{X})}{\partial \mathbf{X}}-\phi^{\mathrm{T}} \frac{\partial \mathbf{K}(\mathbf{X})}{\partial \mathbf{X}} \mathbf{u}$

where $\phi$ is the vector associated to adjoint displacement field, $\frac{\partial \Psi_{m}(\mathbf{u}, \mathbf{X})}{\partial \mathbf{u}}$ represents the adjoint forces vector.

Taking the components of the variance-covariance matrix $\mathbf{C}_{\boldsymbol{\Psi}}$ defined from Eqs. (24)-(26) it is possible to calculate the coefficient of variation as the importance measure of each input parameter on uncertainty propagation of the response function $\Psi_{m}(\mathbf{X})$. The coefficient of variation for the response functional defined in Eq. (15) is

$\mathrm{C} . \mathrm{V} \cdot \bar{u}=\frac{\sqrt{\operatorname{var}(\bar{u})}}{|E\langle\bar{u}\rangle|}=\frac{\sqrt{\operatorname{var}(\bar{u})}}{\bar{u}}$

C.V. $\bar{R}=\frac{\sqrt{\operatorname{var}(\bar{R})}}{|E\langle\bar{R}\rangle|}=\frac{\sqrt{\operatorname{var}(\bar{R})}}{\bar{R}}$

The local importance measure of the uncertainty propagation on response is implemented using two approaches: a group analysis and a joint analysis.

The first analysis is based on grouping the input parameters with uncertainty according to their characteristics. In the present model four groups of input parameters are considered:

- Elastic and strength properties of the laminate $(p)$.

- Ply angle $(a)$.

- Thickness of the laminate $(h)$ and

- Point loads $(F)$ applied on the structure.

The input parameter vectors are denoted by $\mathbf{X}_{p}, \mathbf{X}_{a}, \mathbf{X}_{h}$ and $\mathbf{X}_{F}$ respectively. The coefficients of variation $\mathrm{C} . \mathrm{V} . \bar{u}(\%)$ and C.V. $\bar{R}(\%)$ are obtained within each group considering only the variation of input parameters belonging to the group and keeping the remaining ones without variation.

The second analysis is performed using the joint contribution of all input parameters to study the uncertainty propagation on structural response.
The two types of analysis described above can be implemented using the known variance-based decomposition technique applied to each group of input parameters. However, this decomposition uses the local measure importance. Denoting by $\mathbf{X}_{\mathrm{g}}$ each of the previous groups the corresponding fractional contribution to the variance of $\Psi_{m}$ is written as

$\operatorname{var}\left(\Psi_{m}\left(\mathbf{X}_{\mathrm{g}}\right)\right)=\frac{\sum_{X_{j} \in \mathbf{X}_{\mathrm{g}}}\left(\frac{\partial \Psi_{m}\left(\mathbf{X}^{0}\right)}{\partial X_{j}}\right)^{2} \operatorname{var}\left(X_{j}\right)}{\operatorname{var}\left(\Psi_{m}\right)}$

The above local importance measure is denoted by explained variance and has the following additive property:

$$
\begin{aligned}
& \operatorname{var}\left(\Psi_{m}\left(\mathbf{X}_{p}\right)\right)+\operatorname{var}\left(\Psi_{m}\left(\mathbf{X}_{a}\right)\right)+\operatorname{var}\left(\Psi_{m}\left(\mathbf{X}_{h}\right)\right) \\
& \quad+\operatorname{var}\left(\Psi_{m}\left(\mathbf{X}_{F}\right)\right)=1
\end{aligned}
$$

that is a consequence of the first-order Taylor expansion of structural response function adopted in Eq. (19) and considering non-correlated input parameters.

\section{Global Sensitivity Analysis}

The previously local measures of sensitivity are not enough for a fully evaluation of the influence of input parameters on structural response uncertainty $[16,17]$. The evaluation of the uncertainty propagation on response in the neighbourhood of mean values of input parameters is limitative. To analyse the influence of individual parameters and parameter groups on the uncertainty at the output structural response $\Psi_{m}$ Global Sensitivity Analysis (GSA) techniques must be used. Global Sensitivity Analysis denotes the set of methods that consider the whole variation range of inputs and tries to share the output response uncertainty among the input parameters.

\subsection{Global variance-based method}

Among GSA techniques the variance-based methods are the most appropriated [17]. However its application for composite structures is complex and expensive from the computational point of view. In this work the variancebased methods is applied to a group of input parameters namely the physical properties of composites and then compared with local importance measures.

Assuming that $\mathbf{X}=\left(X_{1}, \ldots, X_{n}\right)$ are $n$ independent input parameters and $\Psi_{m}$ is the model function previously defined, an indicator of the importance of an input parameter $X_{i}$ could be based on the outcomes of the variance of $\Psi_{m}$ if $X_{i}$ is fixed at its true value $x_{i}^{*}: \operatorname{var}\left(\Psi_{m} \mid X_{i}=x_{i}^{*}\right)$. This is the conditional variance of $\Psi_{m}$ given $X_{i}=x_{i}^{*}$. However, in most cases the true value $x_{i}^{*}$ of $X_{i}$ is not known and then to overcome this difficulty the average of the conditional variance under all possible values for $X_{i}$ denoted by $\mathrm{E}\left\langle\operatorname{var}\left(\Psi_{m} \mid X_{i}\right)\right\rangle$, is calculated. Considering the following algebraic property: 


$$
\operatorname{var}\left(\Psi_{m}\right)=\operatorname{var}\left(E\left\langle\Psi_{m} \mid X_{i}\right\rangle\right)+E\left\langle\operatorname{var}\left(\Psi_{m} \mid X_{i}\right)\right\rangle
$$

the variance of the conditional expectation $\operatorname{var}\left(E\left\langle\Psi_{m} \mid X_{i}\right\rangle\right)$ can be used as an indicator of the importance of $X_{i}$ on the variance of $\Psi_{m}$. This indicator is directly proportional to the importance of $X_{i}$. A normalised index can be established using the conditional expectation as

$S_{i}=\frac{\operatorname{var}\left(E\left\langle\Psi_{m} \mid X_{i}\right\rangle\right)}{\operatorname{var}\left(\Psi_{m}\right)}$

named first-order sensitivity index by Sobol [11]. Furthermore, Sobol proposed a complete variance decomposition of the uncertainty associated with $\Psi_{m}$ into components depending on individual parameters and interactions between individual parameters. This procedure explains the variance $\operatorname{var}\left(\Psi_{m}\right)$ as a contribution of the partial variance associated to each individual parameters or each parameter groups as

$\operatorname{var}\left(\Psi_{m}\right)=\sum_{i} V_{i}+\sum_{i<j} V_{i j}+\sum_{i<j<k} V_{i j k}+\cdots+V_{12 \ldots n}$

where

$$
\begin{aligned}
V_{i}= & \operatorname{var}\left(E\left\langle\Psi_{m} \mid X_{i}\right\rangle\right) \\
V_{i j}= & \operatorname{var}\left(E\left\langle\Psi_{m} \mid X_{i}, X_{j}\right\rangle\right)-V_{i}-V_{j} \\
V_{i j k}= & \operatorname{var}\left(E\left\langle\Psi_{m} \mid X_{i}, X_{j}, X_{k}\right\rangle\right)-V_{i j} \\
& \quad-V_{j k}-V_{i k}-V_{i}-V_{j}-V_{k}
\end{aligned}
$$

and assuming that all input parameters are independent in this approach. From this decomposition higher-order sensitivity indices can be established in particular the second-order sensitivity index as:

$S_{i j}=\frac{V_{i j}}{\operatorname{var}\left(\Psi_{m}\right)}$

The second-order index $S_{i j}$ defines the sensitivity of the structural response $\Psi_{m}$ to the interaction between $X_{i}$ and $X_{j}$, i.e. the portion of the variance of $\Psi_{m}$ that is not included in the individual effects of $X_{i}$ and $X_{j}$. The sum of all order indices is equal to 1 in case all input parameters are independent.

It should be referred that all terms in Eq. (36) involving more than one input parameter in the summation are equal to zero if $\Psi_{m}$ has the additive property:

$\Psi_{m}(\mathbf{X})=\sum_{i=1}^{n} \varphi\left(X_{i}\right)$

In this case $\operatorname{var}\left(\Psi_{m}\right)=\sum_{i} V_{i}$ and the importance of the input parameters with respect to response uncertainty is given by $S_{i}$. An example of the additive property is the approximation model of Eq. (20) based on Taylor expansion. However this model is only valid in a neighbourhood of nominal values of input parameters. In general it is expected that $\Psi_{m}$ is not additive relatively to the input parameters and some interactions between parameters.

\subsection{GSA evaluation using Monte Carlo simulation}

An exhaustive sensitivity analysis should provide the estimation of first and second-order indices. One of the problems using global sensitivity indices is the computational cost. Due to the large number of input parameters in the uncertainty propagation analysis on composite structures, Finite Element Method evaluations become very expensive. In this work the Monte Carlo simulations is used for the estimation of GSA indices. To reduce the computational costs the analysis is implemented using groups of input parameters and considering only the first-order sensitivity index.

The proposed methodology is based on the following algorithm:

1. Let us consider $p$ groups of non-correlated input parameters $\pi=\left(\pi_{1}, \ldots, \pi_{p}\right)$ following a normal distribution $N$ with mean $\bar{\pi}_{i}$ and standard deviation $\sigma_{i}$ represented by $\pi_{i} \sim N\left(\bar{\pi}_{i}, \sigma_{i}\right)$.

2. Considers a set of random numbers, $\Gamma_{f i x}=$ $\left(\lambda_{1}, \ldots, \lambda_{N_{f}}\right)$, following a standard normal distribution $N(0,1)$. These random numbers are used to generate the fixed values of the input parameter $\pi_{i}$ :

3. For each input parameter $\pi_{j \neq i}$ a sample matrix is generated by independently collecting samples of $(p-1)$ random numbers following a normal distribution $N(0,1)$ :

$$
\mathbf{M}_{\alpha}=\left[\begin{array}{ccc}
\alpha_{1,1} & \ldots & \alpha_{1, p-1} \\
\vdots & \ddots & \vdots \\
\alpha_{N_{r}, 1} & \cdots & \alpha_{N_{r, p}-1}
\end{array}\right]
$$

where the size of the sample is $N_{r}$.

4. Repeat for each input parameter $\pi_{i}, i=1 \rightarrow p$

Do $k=1 \rightarrow N_{f}$

Do $q=1 \rightarrow N_{r}$

$\pi_{j}^{k, q}=\left\{\begin{array}{ll}\bar{\pi}_{j}+\lambda_{k} \sigma_{j} & \text { if } j=i \\ \bar{\pi}_{j}+\alpha_{q, j} \sigma_{j} & \text { if } j \neq i\end{array}\right.$ for $j=1, \ldots, p$

Evaluation of the structural response: $\Psi_{m}\left(\pi^{k, q}\right)$, being the vector $\pi^{k, q}$ nominal values of $\pi$, with components $\pi_{j}^{k, q}$.

\section{End Do}

Estimate the conditional expectation of structural response function $\Psi_{m}$ by

$E\left\langle\Psi_{m} \mid \pi_{i}\right\rangle \approx \bar{\Psi}_{m}^{k}=\frac{1}{N_{r}} \sum_{q=1}^{N_{r}} \Psi_{m}\left(\pi^{k, q}\right)$

\section{End Do}

Estimate of the mean values

$\overline{\bar{\Psi}}_{m}=\frac{1}{N_{f}} \sum_{k=1}^{N_{f}} \bar{\Psi}_{m}^{k}$

Estimation of the variance of the conditional expectation of structural response, fixing the input parameter $\pi_{i}$ : 
$\operatorname{var}\left(E\left\langle\Psi_{m} \mid \pi_{i}\right\rangle\right) \approx \frac{1}{N_{f}-1} \sum_{k=1}^{N_{f}}\left(\bar{\Psi}_{m}^{k}-\overline{\bar{\Psi}}_{m}\right)^{2}$

\section{End repeat}

5. Estimation of variance of structural response $\operatorname{var}\left(\Psi_{m}\right)$ considering the previous $N_{T}=N_{r} \times N_{f} \times p$ simulations for $\Psi_{m}$ :

$$
\begin{aligned}
& E\left\langle\Psi_{m}\right\rangle=\frac{1}{N_{T}} \sum_{i=1}^{p} \sum_{k=1}^{N_{f}} \sum_{q=1}^{N_{r}}\left[\Psi_{m}\left(\pi^{k, q}\right)\right]_{i} \\
& \operatorname{var}\left(\Psi_{m}\right)=\frac{1}{N_{T}-1} \sum_{i=1}^{p} \sum_{k=1}^{N_{f}} \sum_{q=1}^{N_{r}}\left\{\left[\Psi_{m}\left(\pi^{k, q}\right)\right]_{i}-E\left\langle\Psi_{m}\right\rangle\right\}^{2}
\end{aligned}
$$

6. Calculation of the global sensitivity index:

$$
S_{i}=\frac{\operatorname{var}\left(E\left\langle\Psi_{m} \mid \pi_{i}\right\rangle\right)}{\operatorname{var}\left(\Psi_{m}\right)} \quad i=1, \ldots, p
$$

\section{Global variance from local measure: an approach}

The local analysis presented in Section 3 and defined by Eq. (25) based on the evaluation of the variance-covariance matrix of output response functions $\Psi$ is valid only at the neighbourhood of the nominal values and the exact value is obtained in case of linear behaviour. However, this local analysis can be extended to all nominal values belonging to the domain of input parameters. For this, the following approach for uncertainty analysis is applied:

1. Firstly, let us consider $p$ groups of non-correlated input parameters $\pi=\left(\pi_{1}, \ldots, \pi_{p}\right)$ following a normal distribution $N$ with mean $\bar{\pi}_{i}$ and standard deviation $\sigma_{i}$ represented by $\pi_{i} \sim N\left(\bar{\pi}_{i}, \sigma_{i}\right)$. The $k$ th vector of nominal values of $\pi$ is denoted by $\pi_{k}^{0}=\left(\pi_{k, 1}^{0}, \ldots, \pi_{k, p}^{0}\right)$.

2. Consider a sample matrix $\mathbf{M}_{\pi^{0}}$ of nominal values generated by independently sampling of $p$ random numbers following a normal distribution $\pi_{k, i}^{0} \sim N\left(\bar{\pi}_{i}, \sigma_{i}\right)$ :

$$
\mathbf{M}_{\pi^{0}}=\left[\begin{array}{ccc}
\pi_{1,1}^{0} & \cdots & \pi_{1, p}^{0} \\
\vdots & \ddots & \vdots \\
\pi_{N_{\mathrm{s}}, 1}^{0} & \cdots & \pi_{N_{\mathrm{s}}, p}^{0}
\end{array}\right]
$$

3. Evaluate the variance-covariance matrix at the $k$ th vector of nominal values of $\pi$ as follows:

$$
\begin{aligned}
\mathbf{C}_{\boldsymbol{\Psi}}^{k} & \cong E\left\langle\left(\boldsymbol{\Psi}(\boldsymbol{\pi})-\boldsymbol{\Psi}\left(\boldsymbol{\pi}_{k}^{0}\right)\right)\left(\boldsymbol{\Psi}(\boldsymbol{\pi})-\boldsymbol{\Psi}\left(\boldsymbol{\pi}_{k}^{0}\right)\right)^{\mathrm{T}}\right\rangle \text { for } k \\
& =1, \ldots, N_{\mathrm{s}}
\end{aligned}
$$

or according to Eq. (26),

$$
\mathbf{C}_{\boldsymbol{\Psi}}^{k}=\left[\begin{array}{cc}
\operatorname{var}(\bar{u})_{k} & \operatorname{cov}(\bar{R}, \bar{u})_{k} \\
\operatorname{cov}(\bar{u}, \bar{R})_{k} & \operatorname{var}(\bar{R})_{k}
\end{array}\right] \cong \mathbf{S}_{k} \mathbf{C}_{x}\left(\mathbf{S}_{k}\right)^{\mathrm{T}}
$$

4. Evaluate the average of the variance of output response functions from the variance-covariance matrix $\mathbf{C}_{\boldsymbol{\Psi}}^{k}$ calculated using the sample matrix $\mathbf{M}_{\pi^{0}}$ of nominal values:

$$
\begin{aligned}
& E(\operatorname{var}(\bar{u}))=\frac{1}{N_{\mathrm{s}}} \sum_{k=1}^{N_{\mathrm{s}}} \operatorname{var}(\bar{u})_{k} \\
& E(\operatorname{var}(\bar{R}))=\frac{1}{N_{\mathrm{s}}} \sum_{k=1}^{N_{\mathrm{s}}} \operatorname{var}(\bar{R})_{k}
\end{aligned}
$$

\section{Numerical studies}

\subsection{Local importance measures}

Aiming to study the influence of input parameter deviations on the structural response based on local importance measure, a numerical example using composite laminated materials is presented in this section. A clamped cylindrical shell laminate structure is considered to test the proposed approach as shown in Fig. 1. Nine vertical loads of mean value $P_{k}=7 \mathrm{kN}$ are applied along the free linear side $(\mathrm{AB})$ of the structure. This free linear side $(\mathrm{AB})$ is constrained in $y$-axis direction.

The structure is divided in four macro-elements grouping all elements and there is one laminate per each macro-element. The laminate distribution on structure is shown in Fig. 1. A common type of composite laminate is the angle-ply configurations [14] without bendingstretching effects due to the symmetry plan. In this investigation the balanced angle-ply laminates with eight layers and the stacking sequence $[+a /+a /-a /-a]_{\mathrm{s}}$ are considered in the symmetric construction. The ply angle $a$ is referred to the $x$-axis of the referential detailed in Fig. 1. All laminates have the same mean thickness of $h_{k}=0.02 \mathrm{~m}$.

The use of multiple materials in composite laminates construction is adopted for the shell structure of the present example. Three composite systems are considered for the material composition of laminates on structure:

- GFRP, E-glass/epoxy (1): The composite system of epoxy resin reinforced by unidirectional fibres of E-glass known as Scotchply 1002 [14] with a fibre volume fraction of 0.450 is adopted for all laminates.

- CFRP, T300/N5208 (2): The composite system of epoxy resin reinforced by unidirectional Carbon fibres with a fibre volume fraction of 0.700 is adopted for all laminates;

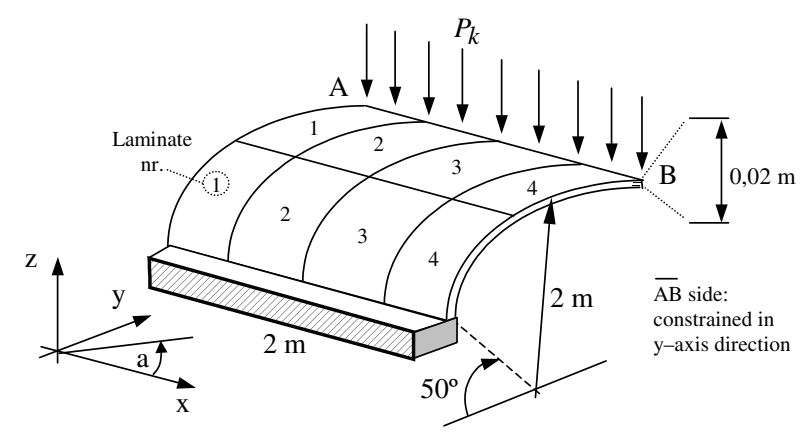

Fig. 1. Cylindrical shell and angle-ply composite laminates distribution (ply angle $a$ referred to $x$-axis). 
- GFRP (1) \& CFRP (2): The use of E-glass/epoxy (1) composite system for laminates number 1 and 4 (see Fig. 1) and Carbon/epoxy T300/N5208 (2) composite system for laminates number 2 and 3 on structure.

The mean values of the elastic and strength properties of the ply material used in the laminate construction of the composite structure are presented in Table 1 [14]. The elastic constants of the orthotropic ply are previously defined in Section 2.1. The ply strength properties are the longitudinal strength in tensile $X$ and in compression $X^{\prime}$, the transversal strength in tensile $Y$ and in compression $Y^{\prime}$, and the shear strength $S$.

Fig. 2 presents the behaviour of structural response for absolute value of maximum displacement $\bar{u}$ and critical Tsai number $\bar{R}$ as the ply angle varies from $0^{\circ}$ to $90^{\circ}$. The critical displacement decreases from a maximum absolute value at $0^{\circ}$ reaching at $90^{\circ}$ a value corresponding to 7-9 times smaller than maximum depending on the laminate material. The critical Tsai number increases 5-9 times from a minimum value along the domain of ply angle $a$ of the laminate material. This shows the important influence of anisotropy with consequences on uncertainties propagation. The mixed composite system GFRP (1) \& CFRP (2) exhibits an average maximum displacement between GFRP, E-glass/epoxy and CFRP, T300/N5208. However the critical Tsai number response functional is very similar for GFRP (1) \& CFRP (2) and GFRP but very different for CFRP.

To investigate the uncertainty propagation on response of composite structure the input parameters with uncertainty are grouped as defined in a previous section: mechanical properties group $(p)$, ply-angle group $(a)$, laminate thickness group $(h)$ and vertical point loads group $(F)$. All the input parameters with uncertainty are non-correlated.

The mechanical properties group $(p)$ includes the following uncertainty input parameters: longitudinal Young's modulus $E_{1, j}$, transversal modulus $E_{2, j}$, transversal tensile strength $Y_{j}$ and shear strength $S_{j}$, where subscript denotes the laminate number. The number of mechanical properties considered as input parameters with uncertainty in this analysis is sixteen: $E_{1, j}, E_{2, j}, Y_{j}, S_{j}, j=1, \ldots, 4$. The choice of these properties as input parameters with uncertainty follows the conclusions of the previous parameterised model presented by António et al. [18]. However, the present study can be extended to other input parameters with uncertainty.
Since the balanced angle-ply laminates construction is applied only one input parameter with uncertainty is considered in ply angle group $(a)$.

The laminate thickness group $(h)$ has four input parameters with uncertainty corresponding to each laminate thickness: $h_{j}, j=1, \ldots, 4$.

Finally the vertical point loads group $(F)$ has nine components applied on structure as shown in Fig. 1: $F_{k}$, $k=1, \ldots, 9$.

To study the influence of uncertainties propagation on the structural response variations at input parameters are considered as follows:

- C.V. $(p)=6 \%$ in mechanical properties group $(p)$.

- Standard deviation, $\sigma(a)=5^{\circ}$, in ply angle group $(a)$.

- C.V. $(h)=6 \%$ in laminate thickness group $(h)$.

- C.V. $(F)=6 \%$ in vertical point loads group $(F)$.

where the coefficient of variation of the input parameter $X_{i}$ was defined as

C.V. $\left(X_{i}\right)=\left|\frac{\sigma_{i}}{X_{i}^{0}}\right|$

being $\sigma_{i}$ and $X_{i}^{0}$ the standard deviation and the nominal value of input parameter respectively. In this case the mean values are assumed as nominal values.

The analysis was implemented independently for each group of input parameters using Eq. (24)-(26) and the above uncertainty input conditions inside the referred groups. Figs. 3-6 show results using the coefficient of variation for the absolute value of maximum displacement on the structure $\bar{u}$ and the critical Tsai number $\bar{R}$ given as functions of ply angle $a$.

The general observations assuming the decomposition in groups of input parameters are:

- The amplitude of variations of uncertainty propagation in maximum displacement response along the ply-angle domain is lower than the ones in critical Tsai number response, for all input parameter groups.

- The variations in C.V.u are high over interval $\left[0^{\circ}, 90^{\circ}\right]$ for uncertainties in ply thickness $(h)$ input parameter group for all composite systems. The variations are also high in ply angle $(a)$ input parameter groups for CFRP composite system and the interval $\left[25^{\circ}, 65^{\circ}\right]$ should be avoided for robust design.

Table 1

Mean values of mechanical properties of unidirectional composite layers

\begin{tabular}{|c|c|c|c|c|}
\hline Material & $E_{1}[\mathrm{GPa}]$ & $E_{2}[\mathrm{GPa}]$ & $G_{12}[\mathrm{GPa}]$ & $v$ \\
\hline \multirow[t]{2}{*}{ T300/N5208 E-glass/epoxy (Scotchply 1002) } & 181.0 & 10.3 & 7.17 & 0.28 \\
\hline & 38.6 & 8.27 & 4.14 & 0.26 \\
\hline \multirow[t]{2}{*}{ T300/N5208 E-glass/epoxy (Scotchply 1002) } & $1500 ; 1500$ & $40 ; 246$ & 68 & 1600 \\
\hline & $1062 ; 610$ & $31 ; 118$ & 72 & 1800 \\
\hline
\end{tabular}



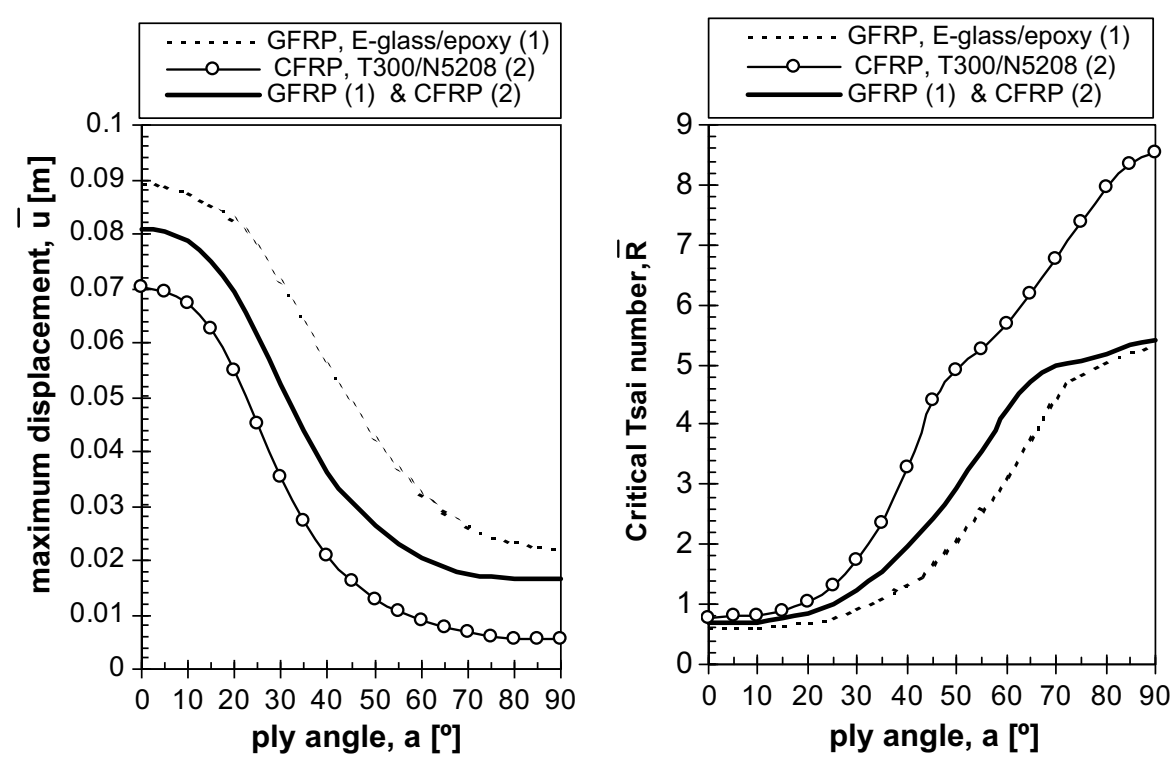

Fig. 2. Influence of anisotropy (ply angle $a$ ) on response functional $\bar{u}$ and $\bar{R}$ for three composite system of laminates used in shell structure.
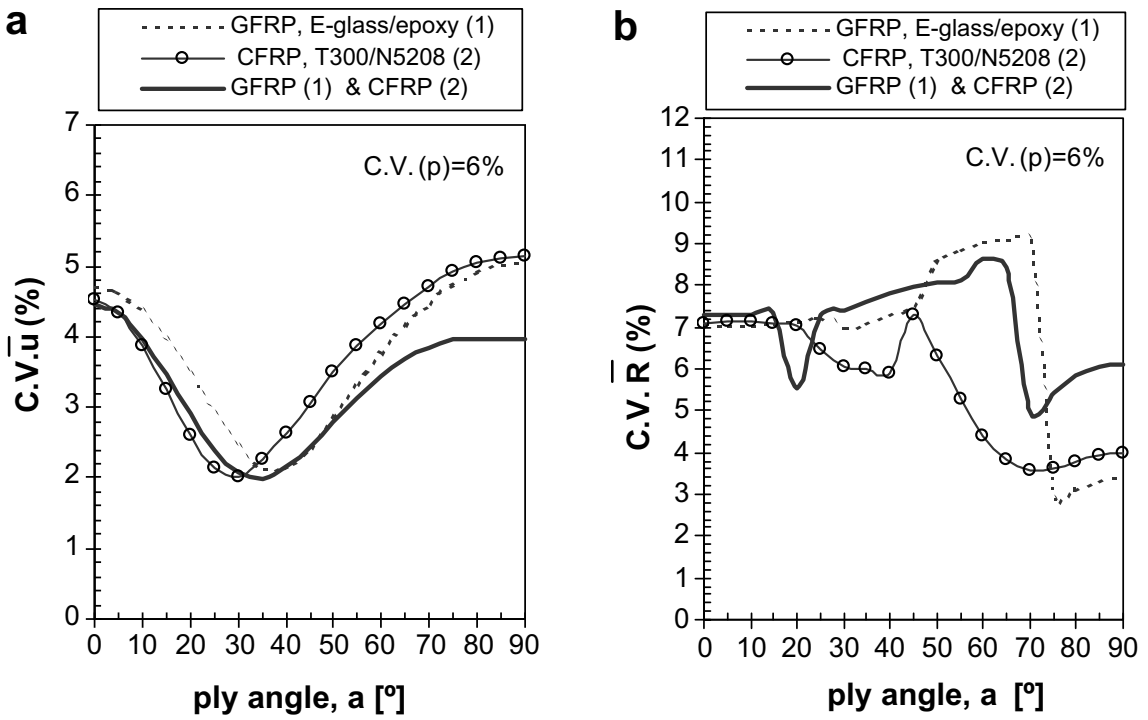

Fig. 3. Coefficient of variation of structural response using Eq. (26), analysis for C.V. $(p)=6 \%$ in mechanical properties group ( $p$ ). (a) Maximum displacement C.V. $\bar{u}(\%)$; (b) critical Tsai number C.V. $\bar{R}(\%)$.

- The variations in C.V. $\bar{R}$ are particularly high for uncertainty in ply angle $(a)$, ply thickness $(h)$ and mechanical properties $(p)$ input parameter groups. In general there is a central zone of the domain that should be avoided for robust design.

- The shape curves of uncertainty propagation in maximum displacement response are similar for all input parameter groups considering the three composite systems. This is related to linear relationship between loads versus displacement existing in equilibrium Eq. (13). For critical Tsai number the shape curves depend strongly on the type of input parameter group and composite system.
- The use of multi-materials as GFRP (1) \& CFRP (2) composite systems has advantages considering the uncertainty propagation in maximum displacement response in particularly for uncertainty in ply angle $(a)$ input parameter group.

Using the variance-covariance matrix $\mathbf{C}_{\boldsymbol{\Psi}}$ presented in Eq. (24) and the definition (26) it is possible to analyse the joint effects of all input parameters for the uncertainties propagation on the structural response. Fig. 7 shows the behaviours of both coefficient of variation C.V. $\bar{u}(\%)$ and C.V.. $\bar{R}(\%)$ as functions of the anisotropy based on ply angle $a$. 

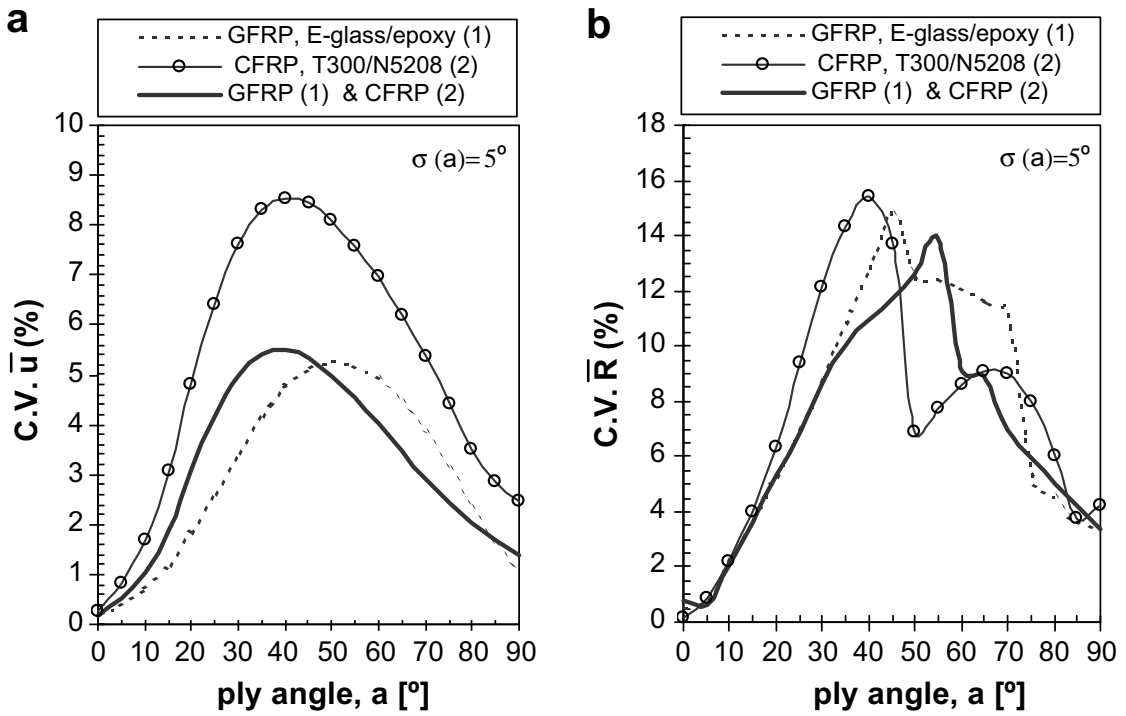

Fig. 4. Coefficient of variation of structural response using Eq. (26), analysis for standard deviation, $\sigma(a)=5^{\circ}$, in ply angle group (a). (a) Maximum displacement C.V.ü(\%); (b) critical Tsai number C.V. $\bar{R}(\%)$.
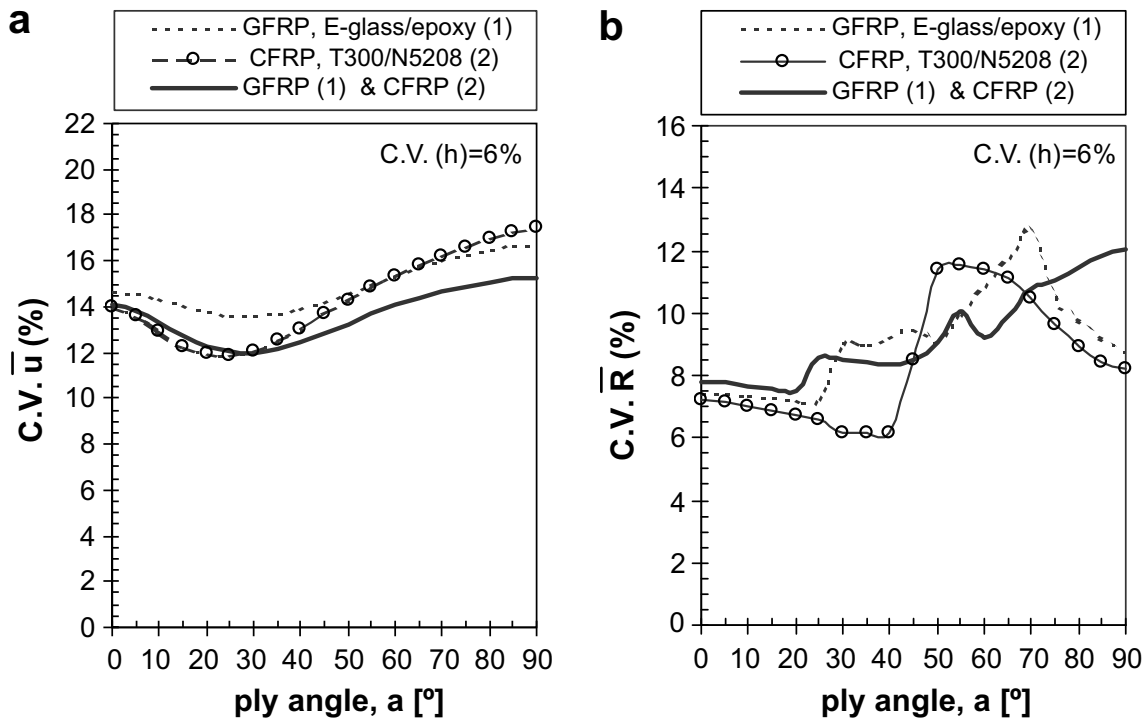

Fig. 5. Coefficient of variation of structural response using Eq. (26), analysis for C.V. $(h)=6 \%$ in laminate thickness group $(h)$. (a) Maximum displacement C.V. $\bar{u}(\%)$; (b) critical Tsai number C.V. $\bar{R}(\%)$.

The general observations considering the joint contribution of all input parameters are:

- The interval of variation of uncertainty propagation on response along the ply angle $a$ domain is $[13 \%, 19 \%]$ for C.V. $\bar{u}(\%)$ and $[10 \%, 20 \%]$ for C.V. $\overline{\mathrm{R}}(\%)$.

- The set of values of ply angle $a$ belonging to the interval $\left[25^{\circ}, 75^{\circ}\right]$ should be avoided due to large coefficient of variation C.V. $\bar{R}(\%)$.

The local importance measure of each input parameter group based on coefficient of variation can be obtained analysing the graphs from Figs. 3-6 compared with the joint effects represented in Fig. 7. The other local importance measure is the explained variance done by Eq. (32).

The explained variance of maximum displacement $\operatorname{var}\left(\overline{\mathrm{u}}\left(\mathrm{X}_{\mathrm{g}}\right)\right)$ and critical Tsai number $\operatorname{var}\left(\overline{\mathrm{R}}\left(\mathrm{X}_{\mathrm{g}}\right)\right)$ both using Eq. (32) based on decomposition by groups and local measures of uncertainty propagation is presented in Figs. 8 and 9. The analysis was implemented for composite system GFRP (1) \& CFRP (2) on structure. It is clear from Fig. 8 that the thickness input parameter group is the most important for uncertainty propagation in maximum displacement response. On other way, the explained variance of critical Tsai number response shows that the most important input parameter group depends on the ply angle $a$ as shown in Fig. 9 . 

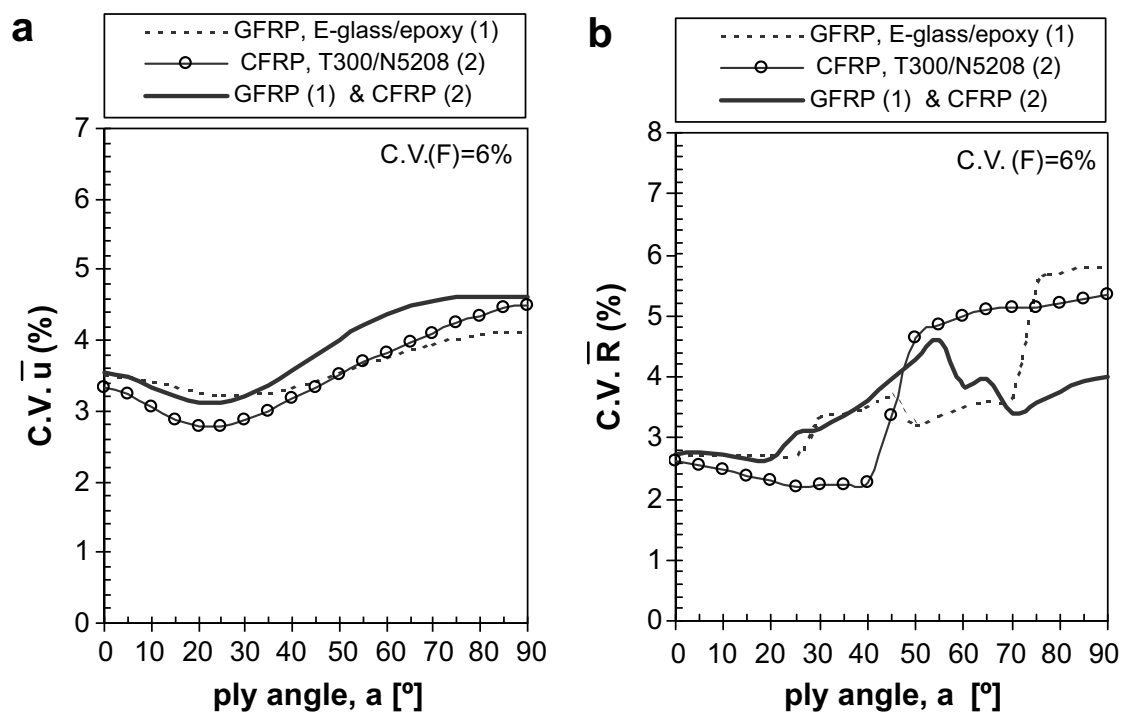

Fig. 6. Coefficient of variation of structural response using Eq. (26), analysis for C.V. $(F)=6 \%$ in vertical point loads $(F)$. (a) Maximum displacement C.V. $\bar{u}(\%)$; (b) critical Tsai number C.V. $\bar{R}(\%)$.
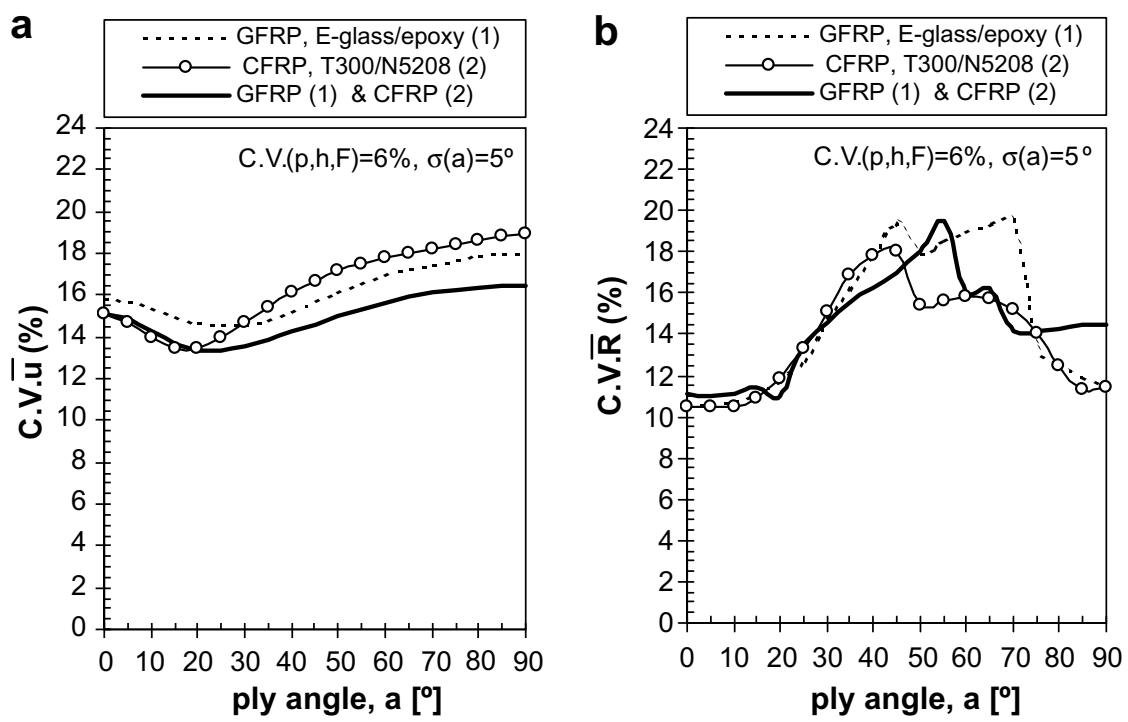

Fig. 7. Coefficient of variation of structural response using Eq. (26), analysis for simultaneous variation of all input groups. (a) Maximum displacement C.V. $\overline{\mathbf{u}}(\%)$; (b) critical Tsai number C.V. $\bar{R}(\%)$.

The previous uncertainty propagation analysis grouping the input parameters and the joint analysis should be driven considering the behaviour of maximum displacement on the structure $\bar{u}$ and the critical Tsai number $\bar{R}$ response functions over the space design of ply angle $a$. The objective is to find a good compromise between structural response values and the minimisation of uncertainty effect on response.

\subsection{Global Sensitivity Analysis (GSA) applications}

In this section the global variance-based method and Monte Carlo simulation proposed in Section 4 is applied to the same shell structure shown in Fig. 1 with all laminates built using the CFRP, T300/N5208 (2) composite sys- tem. Furthermore considering the four laminates distributed on composite structure the mechanical properties are grouped as follows:

- The longitudinal elastic modulus group $E_{1}$ for $E_{1, j}$, $j=1, \ldots, 4$.

- The transversal elastic modulus group $E_{2}$ for $E_{2, j}$, $j=1, \ldots, 4$.

- The transversal strength group $Y$ for $Y_{j}, j=1, \ldots, 4$.

- The shear strength group $S$ for $S_{j}, \mathrm{j}=1, \ldots, 4$.

Then, let us consider the vector of input parameter groups $\pi=\left\{E_{1}, E_{2}, Y, S\right\}$ following a normal distribution $N$ with mean $\bar{\pi}_{i}$ and standard deviation $\sigma_{i}$ represented by 


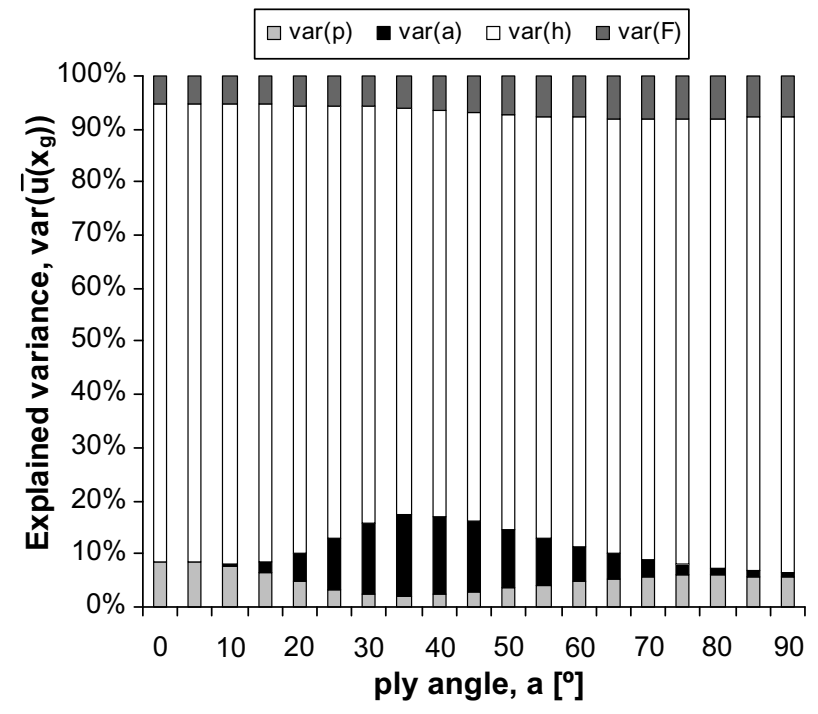

Fig. 8. Explained variance of maximum displacement $\operatorname{var}\left(\bar{u}\left(\mathrm{X}_{\mathrm{g}}\right)\right)$ using decomposition by groups of input parameters based on local importance measure from Eq. (32). Analysis implemented for composite system GFRP (1) \& CFRP (2).

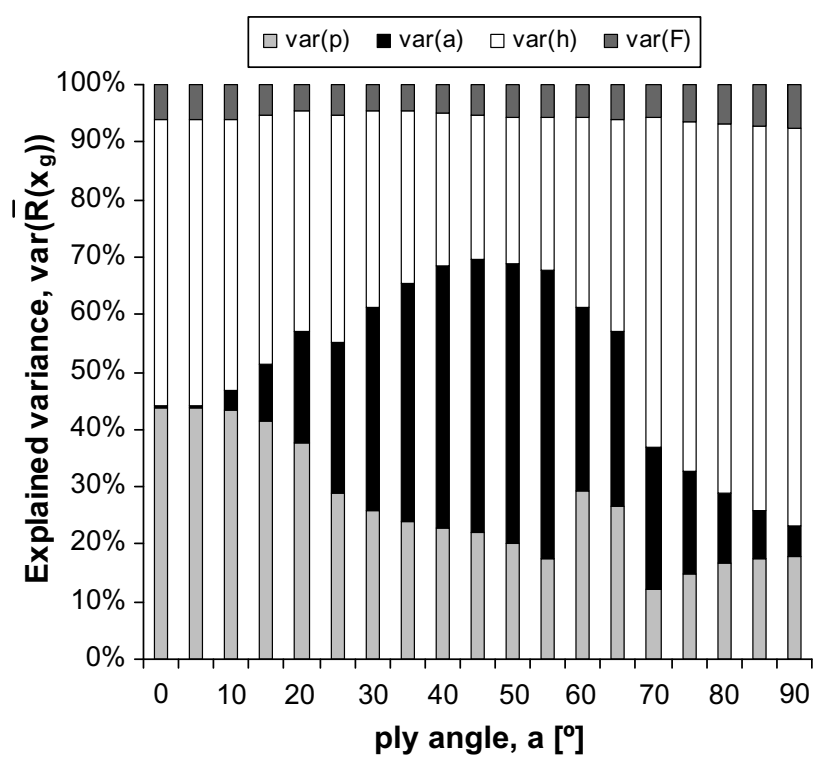

Fig. 9. Explained variance of critical Tsai number $\operatorname{var}\left(\overline{\mathrm{R}}\left(\mathrm{X}_{\mathrm{g}}\right)\right)$ using decomposition by groups of input parameters based on local importance measure from Eq. (32). Analysis implemented for composite system GFRP (1) \& CFRP (2).

$\pi_{i} \sim N\left(\bar{\pi}_{i}, \sigma_{i}\right)$ In particular the statistical values of non-correlated input parameters are:

$$
\begin{aligned}
& E_{1} \sim N(181.000,10.860) \mathrm{GPa} \\
& E_{2} \sim N(10.300,0.618) \mathrm{GPa} \\
& Y \sim N(40.000,2.400) \mathrm{MPa} \\
& S \sim N(6.800,4.080) \mathrm{MPa}
\end{aligned}
$$

The formulation presented in Section 4 is implemented for critical Tsai number response functional $\bar{R}$ and using the above mechanical properties as input parameters. To ex- plain the global variance $\operatorname{var}(\bar{R})$ as a contribution of the partial variance associated to each input parameter group and further to calculated the respective importance measure the global first-order sensitivity index defined in Eq. (35) or (49) is used.

For the Monte Carlo simulation algorithm proposed in Section 4.2 the size samples are defined as follows:

- a set of random numbers, $N_{f}=50$, following a normal distribution $N(0,1)$ to generate the fixed values of input parameters;

- a sample matrix $\mathbf{M}_{\alpha}$ with dimension $N_{r} \times(p-1)=$ $100 \times 3$ to simulate the non-fixed input parameters.

A total of five thousand simulations was considered in Monte Carlo simulations $\left(N_{f} \times N_{r}\right)$ to estimate the variance of conditional expectation of structural response $\operatorname{var}\left(E\left\langle\bar{R} \mid \pi_{i}\right\rangle\right)$ according to Eq. (46). The simulation process is implemented for each input parameter group $\pi_{i}, \mathrm{i}=1, \ldots, 4$ and the global variance $\operatorname{var}(\bar{R})$ can be estimated from the twenty thousand simulations following Eqs. (48) and (49).

An important aspect of the present work is to study the influence of anisotropy in the uncertainty propagation on structural response. Then, GSA is implemented as a function of ply angle $a$. Fig. 10 shows the global variance $\operatorname{var}(\bar{R})$, explained by first-order sensitivity index $S_{i}$ for input parameter groups $\pi=\left\{E_{1}, E_{2}, Y, S\right\}$ and CFRP, T300/ N5208 composite system:

$S_{i}=\frac{\operatorname{var}\left(E\left\langle\bar{R} \mid \pi_{i}\right\rangle\right)}{\operatorname{var}(\bar{R})} \times 100(\%), \quad i=1, \ldots, 4$

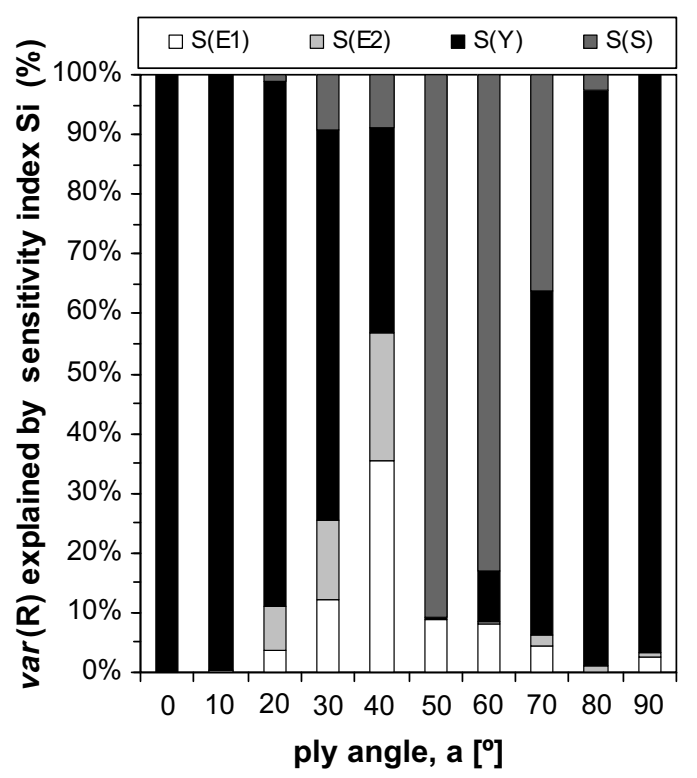

Fig. 10. Global variance $\operatorname{var}(\bar{R})$, explained by first-order sensitivity index $S_{i}$ for input parameters $\pi=\left\{E_{1}, E_{2}, Y, S\right\}$ and CFRP, T300/N5208 composite system. 
Table 2

Ranking of input parameters importance in uncertainty on response functional $\bar{R}$

\begin{tabular}{|c|c|c|c|c|c|c|c|c|c|c|}
\hline \multirow[t]{2}{*}{ Ranking } & \multicolumn{10}{|c|}{ Ply angle $a$} \\
\hline & $0^{\circ}$ & $10^{\circ}$ & $20^{\circ}$ & $30^{\circ}$ & $40^{\circ}$ & $50^{\circ}$ & $60^{\circ}$ & $70^{\circ}$ & $80^{\circ}$ & $90^{\circ}$ \\
\hline 1 st & $Y$ & $Y$ & $Y$ & $Y$ & $E_{1}$ & $S$ & $S$ & $Y$ & $Y$ & $Y$ \\
\hline 2nd & $E_{2}$ & $E_{2}$ & $E_{2}$ & $E_{2}$ & $Y$ & $E_{1}$ & $Y$ & $S$ & $S$ & $E_{1}$ \\
\hline $3 \mathrm{rd}$ & $E_{1}$ & $S$ & $E_{1}$ & $E_{1}$ & $E_{2}$ & $E_{2}$ & $E_{1}$ & $E_{1}$ & $E_{2}$ & $E_{2}$ \\
\hline 4 th & $S$ & $E_{1}$ & $S$ & $S$ & $S$ & $Y$ & $E_{2}$ & $E_{2}$ & $E_{1}$ & $S$ \\
\hline
\end{tabular}

The aim of this modelling is to rank the input parameters according to variance response measure. Input parameters with higher contribution for conditional variance $\operatorname{var}\left(E\left\langle\bar{R} \mid \pi_{i}\right\rangle\right)$ will have higher-sensitivity index $S_{i}$ taken as the global uncertainty importance measure of the input parameter $\pi_{i}$. It is evident from Fig. 10 that the most important input parameter along ply-angle domain is the transversal strength group $Y$ except for a short interval $\left[40^{\circ}, 60^{\circ}\right]$ where the longitudinal elastic modulus $E_{1}$ and shear strength $S$ are important. In Table 2 it is ranked the input parameters importance based on uncertainty propagation on response functional $\bar{R}$ obtained from GSA.

\subsection{Global variance from local measure}

The idea is to test the approach proposed in Section 5 using the shell structure example of Fig. 1 for CFRP, T300/N5208 (2) composite system. The results are obtained considering the same groups of input parameters adopted in the previous section. The sample matrix $\mathbf{M}_{\pi^{0}}$ of nominal values with dimension $N_{\mathrm{s}} \times p=100 \times 4$ was generated based on statistical data defined in Eq. (56). The variance-covariance matrix $\mathbf{C}_{\boldsymbol{\Psi}}^{k}$ defined in Eq. (52) is evaluated for each $k$ th nominal values for non-correlated input parameters. In this simulation it is assumed that the standard deviation doesn't varie for each nominal value of input parameter. Finally the average of variance of output response function $\bar{R}$ is estimated from

$E(\operatorname{var}(\bar{R}))=\frac{1}{N_{\mathrm{s}}} \sum_{k=1}^{N_{\mathrm{s}}} \operatorname{var}(\bar{R})_{k}$

Fig. 11 shows the intervals of variation of $\operatorname{var}(\bar{R})$ over sample matrix $\mathbf{M}_{\pi^{0}}$ of nominal values of input parameter groups $\pi=\left\{E_{1}, E_{2}, Y, S\right\}$. The influence of anisotropy was studied and the results are referred to ply angle $a$ domain $\left[0^{\circ}, 90^{\circ}\right]$. The minimum values are close to average of $\operatorname{var}(\bar{R})$. This means that values above average of $\operatorname{var}(\bar{R})$ are not frequent. The variance of Tsai number response functional is low and doesn't change on interval $\left[0^{\circ}, 30^{\circ}\right]$.

\subsection{Needs for global sensitivity analysis (GSA)}

A comparison between local and global variance measures is established aiming to study the needs for a more expensive uncertainty analysis on response as supported

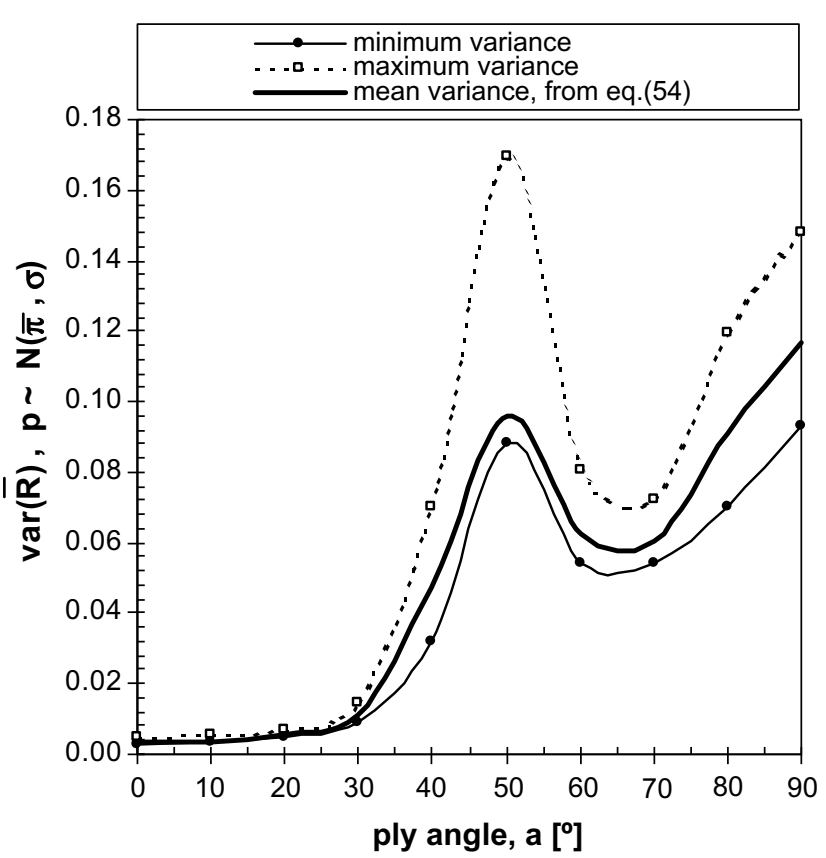

Fig. 11. Global variance $\operatorname{var}(\bar{R})$ from local measure, for input parameters $\pi=\left\{E_{1}, E_{2}, Y, S\right\}$ and composite system CFRP, T300/N5208 (2).

by GSA. Let us consider the example of Fig. 1 using the CFRP, T300/N5208 (2) composite system and the same statistical data defined in Sections 6.2 and 6.3 for input parameter groups $\pi=\left\{E_{1}, E_{2}, Y, S\right\}$. The methodologies discussed previously are compared:

- The variance $\operatorname{var}(\bar{R})$ obtained from local importance measure methodology through Eq. (26) calculated around the nominal values (Section 3).

- Global variance $\operatorname{var}(\bar{R})$ estimated from Monte Carlo method defined in Eqs. (47) and (48) after 20000 simulations.

- The global variance estimated by the average of variance of output response function $\bar{R}$ as defined in Section 5 and Eq. (58).

Fig. 12 shows response variance $\operatorname{var}(\bar{R})$ obtained using the referred methodologies over ply angle $a$ domain. The three curves are close and only diverge shortly in interval $\left[75^{\circ}, 90^{\circ}\right]$. This means that for ply-angle laminates as $[+a /+a /-a /-a]_{s}$, the GSA is not necessary. 


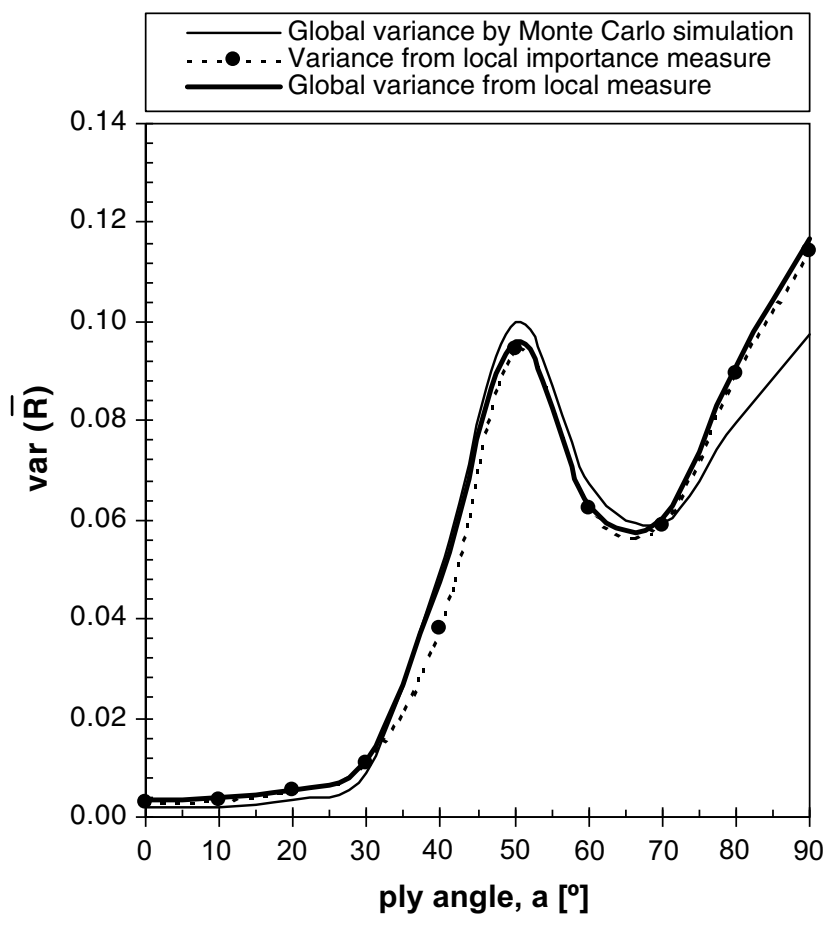

Fig. 12. Comparison of $\operatorname{var}(\bar{R})$ obtained using three different methods, for input parameters $\pi=\left\{E_{1}, E_{2}, Y, S\right\}$ and composite system CFRP, T300/ N5208 (2).

\section{Conclusions}

The uncertainty propagation on structural response of composites was studied in this paper using three different approaches: a first-order local method, a Global Sensitivity Analysis supported by a variance-based method and an extension of local variance to estimate the global variance over all domain of inputs. The objective is to discuss the advantages of less expensive local analysis versus more accurate analysis performed by Global Sensitivity Analysis.

Sample results are shown for shell composite laminated structures built with different composite systems including multi-materials. The importance measures of input parameters on structural response based on numerical results was established and discussed as a function of anisotropy of composite materials. The needs for global variance methods are discussed by comparing the results obtained from proposed methodologies. The results show that a firstorder local method is acceptable to analyse the uncertainty propagation on response for angle-ply laminates. On other hand the validation of results was reached with the performed analysis.
The uncertainty analysis is very useful in designing laminated composite structures minimizing the unavoidable effects of input parameter uncertainties on structural reliability.

\section{References}

[1] Oh DH, Librescu L. Free vibration and reliability of composite cantilevers featuring uncertain properties. Reliab Eng Syst Safety 1997;56:265-72.

[2] Noor AK, Starnes Jr JH, Peters JM. Uncertainty analysis of stiffened composite panels. Compos Struct 2001;51:139-58.

[3] Singh BN, Yadav D, Iyengar NGR. Stability analysis of laminated cylindrical panels with uncertain material properties. Compos Struct 2001;54:17-26.

[4] Adali S, Lene F, Duvaut G, Chiaruttini V. Optimization of laminated composites subject to uncertain buckling loads. Compos Struct 2003;62:261-9.

[5] Walker M, Hamilton R. A technique for optimally designing fibrereinforced laminated plates with manufacturing uncertainties for maximum buckling strength. Eng Optim 2005;37(2):135-44.

[6] Gumbert CR, Newman PA. High-fidelity computational optimization for 3-D flexible wings: Part II - Effect of random geometric uncertainty on design. Optim Eng 2005;6:139-56.

[7] Yang J, Liew KM, Kitipornchai S. Stochastic analysis of compositionally graded plates with randomness under static loading. Int $\mathbf{J}$ Mech Sci 2005;47:1519-41.

[8] Ramroth WT, Krysl P, Asaro RJ. Sensitivity and uncertainty analyses for FE thermal model of FRP panel exposed to fire. Compos Part A: Appl Sci Manufact 2006;37:1082-91.

[9] Helton JC, Davis FJ. Latin hypercube sampling and the propagation of uncertainty in analyses of complex systems. Reliab Eng Syst Safety 2003;81:23-69.

[10] Helton JC, Davis FJ. Survey of sampling-based methods for uncertainty and sensitivity analysis. Reliab Eng Syst Safety 2006;91: 1175-209.

[11] Sobol IM. Sensitivity estimates for nonlinear mathematical models. Math Model Comput Exp 1993;1(4):407-14.

[12] Ahmad S. Curved finite elements in the analysis of solid, shell and plate structures. Ph.D. Thesis, University College of Swansea, UK; 1969.

[13] Figueiras JA. Ultimate load analysis of anisotropic and reinforced concrete plates and shells. Ph.D. Thesis, University College of Swansea, UK; 1983.

[14] Tsai S.W. Composites Design, Dayton, USA, Think Composites; 1987.

[15] Cacuci DG. Theory, vol. 1. Boca Raton, FL: Chapman \& Hall/CTC Press; 2003

[16] Borgonovo E, Apostolakis GE, Tarantola S, Saltelli A. Comparison of global sensitivity analysis techniques and importance measures in PSA. Reliab Eng Syst Safety 2003;79:175-85.

[17] Saltelli A, Ratto M, Tarantola S, Campolongo F. Sensitivity analysis practices: strategies for model-based inference. Reliab Eng Syst Safety 2006;91:1109-25.

[18] Conceição António CA, Torres Marques A, Soeiro AV. Influence of physical properties randomness in laminated composites strength. Proceedings of ICCM-9, Madrid - Spain. University of Zaragoza \& Woodhead Publishing, Ltd; 1993. 